\title{
Molecular insights into the behaviour of bile salts at interfaces: a key to their role in lipid digestion
}

Article

Accepted Version

Creative Commons: Attribution-Noncommercial-No Derivative Works 4.0

Pabois, O., Lorenz, C. D., Harvey, R. D., Grillo, I., Grundy, M. M.-L., Wilde, P. J., Gerelli, Y. and Dreiss, C. A. (2019) Molecular insights into the behaviour of bile salts at interfaces: a key to their role in lipid digestion. Journal of Colloid and Interface Science, 556. pp. 266-277. ISSN 0021-9797 doi: https://doi.org/10.1016/j.jcis.2019.08.010 Available at https://centaur.reading.ac.uk/85434/

It is advisable to refer to the publisher's version if you intend to cite from the work. See Guidance on citing.

To link to this article DOI: http://dx.doi.org/10.1016/j.jcis.2019.08.010

Publisher: Elsevier

All outputs in CentAUR are protected by Intellectual Property Rights law, including copyright law. Copyright and IPR is retained by the creators or other copyright holders. Terms and conditions for use of this material are defined in the End User Agreement. 


\section{CentAUR}

Central Archive at the University of Reading

Reading's research outputs online 


\section{Journal Pre-Proof}

Regular Article

Molecular insights into the behaviour of bile salts at interfaces: a key to their

role in lipid digestion

Olivia Pabois, Christian D. Lorenz, Richard D. Harvey, Isabelle Grillo, Myriam M.-L. Grundy, Peter J. Wilde, Yuri Gerelli, Cécile A. Dreiss

PII:

S0021-9797(19)30911-7

DOI: https://doi.org/10.1016/j.jcis.2019.08.010

Reference: $\quad$ YJCIS 25266

To appear in: $\quad$ Journal of Colloid and Interface Science

Received Date: $\quad 4$ April 2019

Revised Date: $\quad 21$ June 2019

Accepted Date: $\quad 3$ August 2019

Please cite this article as: O. Pabois, C.D. Lorenz, R.D. Harvey, I. Grillo, M. M.-L. Grundy, P.J. Wilde, Y. Gerelli, C.A. Dreiss, Molecular insights into the behaviour of bile salts at interfaces: a key to their role in lipid digestion, Journal of Colloid and Interface Science (2019), doi: https://doi.org/10.1016/j.jcis.2019.08.010

This is a PDF file of an article that has undergone enhancements after acceptance, such as the addition of a cover page and metadata, and formatting for readability, but it is not yet the definitive version of record. This version will undergo additional copyediting, typesetting and review before it is published in its final form, but we are providing this version to give early visibility of the article. Please note that, during the production process, errors may be discovered which could affect the content, and all legal disclaimers that apply to the journal pertain.

(C) 2019 Published by Elsevier Inc. 


\section{Molecular insights into the behaviour of bile salts at interfaces: a key to their role in lipid digestion}

Olivia Pabois $^{\text {a, b}}$, Christian D. Lorenz ${ }^{c}$, Richard D. Harvey ${ }^{d}$, Isabelle Grillo ${ }^{a}$, Myriam M.-L. Grundy ${ }^{\mathrm{e}}$, Peter J. Wildef, Yuri Gerellia*, Cécile A. Dreiss ${ }^{\mathrm{b}^{*}}$

a Institut Laue-Langevin, Grenoble 38000, France

${ }^{b}$ School of Cancer and Pharmaceutical Sciences, Institute of Pharmaceutical Science, King's College London, London SE1 9NH, United-Kingdom

' Department of Physics, King's College London, London WC2R 2LS, United-Kingdom

d Institut für Pharmazie, Martin-Luther-Universität Halle-Wittenberg, Halle (Saale) 06099, Germany

e School of Agriculture, Policy and Development, University of Reading, Reading RG6 6AR, United-Kingdom

${ }^{f}$ Quadram Institute Bioscience, Norwich Research Park, Norwich NR4 7UA, United-Kingdom

E-mail addresses:

olivia.pabois@kcl.ac.uk; chris.lorenz@kcl.ac.uk; $\underline{\text { richard.harvey@pharmazie.uni-halle.de; }}$ grillo@ill.fr; m.m.grundy@reading.ac.uk; peter.wilde@quadram.ac.uk; gerelli@ill.fr; cecile.dreiss@kcl.ac.uk

Corresponding authors:

Cécile A. Dreiss:

King's College London

School of Cancer \& Pharmaceutical Sciences

Franklin-Wilkins Building

150 Stamford Street

SE1 9NH London, UK

Tel: +44 (0)2078483766

Yuri Gerelli:

Institut Max von Laue - Paul Langevin 
71 avenue des Martyrs

38000 Grenoble, France

Tel: +33 (0)4 76207068 


\section{Abstract}

Hypotheses. Understanding the mechanisms underlying lipolysis is crucial to address the ongoing obesity crisis and associated cardiometabolic disorders. Bile salts (BS), biosurfactants present in the small intestine, play key roles in lipid digestion and absorption. It is hypothesised that their contrasting functionalities - adsorption at oil/water interfaces and shuttling of lipolysis products away from these interfaces - are linked to their structural diversity. We investigate the interfacial films formed by two BS, sodium taurocholate (NaTC) and sodium taurodeoxycholate (NaTDC), differing by the presence or absence of a hydroxyl group on their steroid skeleton.

Experiments. Their adsorption behaviour at the air/water interface and interaction with a phospholipid monolayer - used to mimic a fat droplet interface - were assessed by surface pressure measurements and ellipsometry, while interfacial morphologies were characterised in the lateral and perpendicular directions by Brewster angle microscopy, X-ray and neutron reflectometry, and molecular dynamics simulations.

Findings. Our results provide a comprehensive molecular-level understanding of the mechanisms governing BS interfacial behaviour. NaTC shows a higher affinity for the air/water and lipid/water interfaces, and may therefore favour enzyme adsorption, whereas NaTDC exhibits a higher propensity for desorption from these interfaces, and may thus more effectively displace hydrolysis products from the interface, through dynamic exchange.

\section{Keywords}

\section{Bile salts; lipid digestion; interfacial properties; DPPC monolayer; neutron reflectometry}

\section{Graphical abstract}
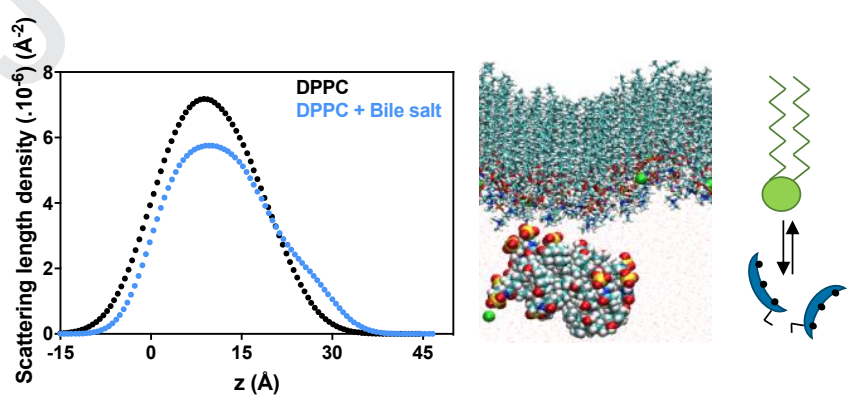

Phospholipid Bile salt 


\section{Introduction}

Over the past few years, changes in both lifestyle and eating habits have resulted in a global increase in obesity prevalence. More specifically, since 1975, worldwide obesity levels have nearly tripled [1]. Dietary fat (lipid) is an excellent source of energy, which is essential for many physiological functions, but its excessive consumption (particularly of saturated fats) contributes towards a large number of chronic cardiovascular illnesses [2]. There is, therefore, an urgent need to develop preventive and therapeutic strategies to tackle this ongoing health crisis. One proposed strategy towards regulating the digestion of lipids consists of using novel foods with appetite-suppressing or satiety-enhancing properties [3]. This approach requires a detailed understanding of the mechanisms involved in fat digestion.

The process of lipid digestion [4,5], also called lipolysis or lipid hydrolysis, requires two water-soluble enzymes (i.e., gastric and pancreatic lipases) that bind to fat droplets and act at the lipid/water interface [6,7]. Dietary triacylglycerols are mostly hydrolysed in the small intestine by co-lipase-dependent pancreatic lipase; products of the lypolysis (diacylglycerols, monoacylglycerols and free fatty acids) accumulate at the lipid droplet interface because of their amphiphilicity $[7,8]$. As a result, they would gradually hinder further enzyme adsorption onto the droplet surface $[7,9]$, if this was not prevented by the presence of bile salts (BS).

BS [10], produced in the liver and stored in the gall bladder, play key roles in promoting lipid digestion and absorption [5,11]. Comprising a short and flexible ionic chain linked to a steroid skeleton [12], they display an unusual planar polarity [13], adsorbing at interfaces and facilitating the adsorption of the co-lipase-dependent pancreatic lipase onto a BS-dominated surface [14-17]. In addition, BS prevent lipase inhibition caused by the accumulation of hydrolysis products by removing polar lipids from the fat droplet surface, solubilising them into mixed micelles [10]. Therefore, BS play two very different roles in fat digestion [5,11]: on the one hand, they promote enzyme-catalysed lipolysis by facilitating the adsorption of the lipase/co-lipase complex at the lipid/water interface, and on the other, they desorb insoluble lipolysis products from the interface, shuttling them into mixed micelles to the gut mucosa, where they are absorbed.

While BS contrasting functionalities have been known for some time, their origin is not well understood. For this reason, the characterisation of their behaviour at both the air/water [18] and lipid/water [19] interfaces has recently begun to garner interest. A recent study [19], 
in particular, has linked differences in BS interfacial properties to their structure, specifically the bile acid moiety: cholate-based BS were found to strongly adsorb onto hydrophobic surfaces (C18-modified silicon oxide sensors), while deoxycholate and chenodeoxycholate derivatives readily desorbed from the interface. Further studies are thus needed to elucidate how BS structure influences their interfacial behaviour and how, in turn, this correlates to their different roles in lipolysis.

This work reports a detailed investigation of the interfacial properties of two selected BS, sodium taurocholate (NaTC) and sodium taurodeoxycholate (NaTDC) (Figure 1), using a range of complementary techniques. These two BS differ in the structure of their bile acid region ( $\mathrm{NaTC}$ has an additional hydroxyl group (Figure 1)), and have previously been shown to display contrasting interfacial behaviour [19]. In this work, we investigate the adsorption/desorption dynamics of BS at the bare air/water interface, using a Langmuir trough (LT) and ellipsometer, and characterise the structure of the interfacial films on a range of length scales, using Brewster angle microscopy (BAM) and X-ray reflectometry (XRR). As a first step towards a more physiologically relevant oil/water interface, we then explore the interaction of BS with a lipid monolayer (1,2-dipalmitoyl-sn-glycero-3-phosphocholine, DPPC) (Figure S1A) deposited at the air/water interface, which mimics the phospholipids present on the surface of lipid droplets [5,9,11,20-22], either as endogenous lipids secreted in the gastrointestinal tract $[4,10]$ or as emulsifying agents present in foodstuffs $[23,24]$. This study provides a molecular-level characterisation of the interfacial films, using neutron reflectometry (NR) combined to molecular dynamics (MD) simulations, bringing an unprecedented insight into the different roles played by BS during lipid digestion. 


\section{Experimental section}

\section{Materials}

DPPC (Figure S1A) and 1,2-dipalmitoyl-d $\mathrm{d}_{62}-\mathrm{sn}$-glycero-3-phosphocholine-1,1,2,2-d $\mathrm{d}_{4}-\mathrm{N}, \mathrm{N}, \mathrm{N}-$ trimethyl- $d_{9}\left(d_{75}-\right.$ DPPC) (Figure S1B) were provided by Avanti Polar Lipids, Inc. (Alabaster, AL, USA), and chloroform $\left(\mathrm{CHCl}_{3}\right)$ by Fisher Scientific (Loughborough, UK). NaTC (P97.0\% TLC) (Figure 1A), NaTDC (P95.0\% TLC) (Figure 1B), sodium glycodeoxycholate (NaGDC, P97.0\% HPLC) (Figure S2) and ethanol (EtOH, P99.8\% GC) were all purchased from Sigma-Aldrich (Gillingham, UK). Ultrapure water, or MilliQ-grade water $\left(\mathrm{H}_{2} \mathrm{O}, 18.2 \mathrm{M} \Omega \cdot \mathrm{cm}\right.$, Merck Millipore, Molsheim, France), was used in all experiments, except for the NR measurements, where deuterium oxide $\left(D_{2} \mathrm{O}, \mathrm{P} 99.9 \%\right)$, provided by Euriso-top SAS (St. Aubin, France), and aircontrast-matched water (ACMW, 8.1\% $\mathrm{D}_{2} \mathrm{O} / 91.9 \% \mathrm{H}_{2} \mathrm{O}$ by volume) were employed. All reagents were used as supplied.

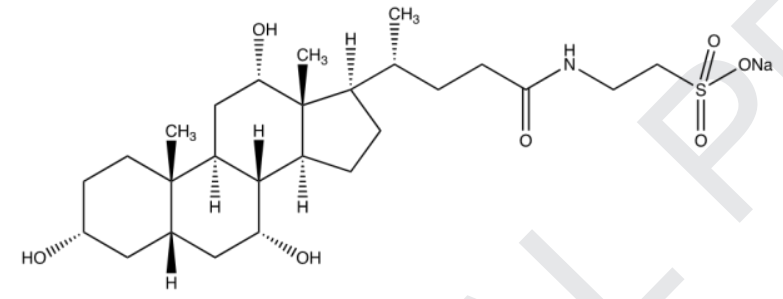

A

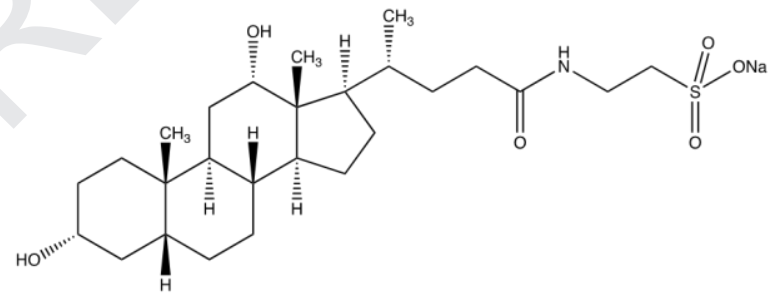

B

Figure 1: Structures of $\operatorname{NaTC}(\mathrm{A})$ and $\mathrm{NaTDC}(\mathrm{B})$. The structure of NaGDC is shown in Figure S2.

\section{Methods}

\section{Langmuir trough (LT) measurements}

Interfacial tension measurements were performed using two different set-ups. The first setup was employed to carry out measurements at a fixed area and with stirring, in a 50-mmdiameter perfluoroalkoxy petri dish (19.6 $\mathrm{cm}^{2}$ surface area and $20 \mathrm{~mL}$ volume of subphase), which was placed over a magnetic stirring plate and used as a trough to study both BS adsorption at the air/water interface and BS interaction with a DPPC monolayer. The second set-up was a classic polytetrafluoroethylene trough (Nima 611D, Nima Technology Ltd, Coventry, UK) of $30 \times 20 \times 0.5 \mathrm{~cm}$ (length $\mathrm{x}$ width $\mathrm{x}$ depth), with a $300 \mathrm{~mL}$ volume of subphase, and was used to measure the surface pressure - area $(\pi-A)$ isotherm of a lipid monolayer at 
the air/water interface. All experiments were carried out at a constant temperature of $23 \pm 2$ ${ }^{\circ} \mathrm{C}$ (room temperature). The surface pressure $(\pi)$ was measured by a Wilhelmy plate, made of chromatographic paper (Whatman International Ltd, Maidstone, UK) of $2.3 \times 1.0 \mathrm{~cm}$ (length $\mathrm{x}$ width) and attached to a calibrated Nima PS4 microbalance.

Prior to any measurement, the trough was thoroughly cleaned with $\mathrm{EtOH}$ and $\mathrm{CHCl}_{3}$ to remove organic impurities, and then filled with ultrapure water (subphase). Surface-active contaminants, dust and bubbles were all removed from the subphase by suction with a pump. The subphase was considered as clean when changes in surface pressure did not exceed \pm 0.2 $\mathrm{mN} / \mathrm{m}$ over approximately two minutes with the petri dish, or when compressed over the entire compression range $\left(64-567 \mathrm{~cm}^{2}\right)$ with the LT.

BS adsorption at the air/water interface. Using a $1 \mathrm{~mL}$ syringe (Becton Dickinson, Madrid, Spain) fitted with a $19 \mathrm{G} \times 1 \frac{1}{2}$ in. needle (Becton Dickinson, Dublin, Ireland), a defined amount of pure BS solution in ultrapure water was injected into the subphase of the petri dish, under constant stirring. Surface pressure $(\pi)$ was measured over time, until it reached a plateau. Each experiment was repeated twice; either a representative curve or an average measurement is shown.

Surface pressure - area $(\pi-A)$ isotherm of lipid monolayers at the air/water interface. A 1 $\mathrm{mg} / \mathrm{mL}$ solution of hydrogenated (DPPC) or deuterated ( $\mathrm{d}_{75}$-DPPC) lipid was prepared in pure $\mathrm{CHCl}_{3}$. For each isotherm, a specific amount ( $45 \mu \mathrm{L}$ for DPPC, $60 \mu \mathrm{L}$ for $\mathrm{d}_{75}$-DPPC) was deposited dropwise onto the aqueous surface, using a $50 \mu \mathrm{L}$ syringe (Hamilton Company, Bonaduz, Switzerland), with the barrier opened at its maximum. After solvent evaporation and monolayer equilibration (ca. 10 minutes), the phospholipid film was compressed at a rate of $35 \mathrm{~cm}^{2} / \mathrm{min}$. Changes in surface pressure $(\pi)$ were recorded as a function of the area per molecule until the lipid monomolecular layer had reached its collapse point, at its collapse surface pressure ( $\left.\pi_{\text {collapse }}\right)$. The average of three isotherms is shown for each lipid.

BS interaction with a DPPC monolayer at the air/water interface. A DPPC monolayer was first formed onto the clean water surface in the petri dish set-up, at the target surface pressure $\left(\pi_{\mathrm{DPPC}}=25 \pm 2 \mathrm{mN} / \mathrm{m}\right)$. After solvent evaporation and film equilibration (ca. 1 hour), stirring was started (at low speed), and a defined amount of pure BS solution in ultrapure water was injected beneath the phospholipid monolayer. The corresponding changes in surface pressure 
$\left(\Delta \pi\right.$, with $\left.\Delta \pi(t)=\pi(t)-\pi_{\mathrm{DPPC}}\right)$ were recorded over time. Each experiment was repeated twice; either a representative curve or the average measurement is shown.

\section{Ellipsometry}

BS adsorption at the air/water interface and interaction with the DPPC monolayer at the air/water interface were further investigated by ellipsometry (Beaglehole Instruments, Wellington, New Zealand). Time-dependent measurements were performed with a 632.8-nmwavelength laser hitting the surface at an incident angle of $50^{\circ}$. In this configuration, changes in the polarisation of light reflected by the interface are measured, over the $1 \mathrm{~mm}^{2}$ area and $1 \mu \mathrm{m}$ depth probed by the laser beam; these changes can be correlated to the amount of material adsorbed at this interface over time. The polarisation state of the incident light is composed of an $s$ - and $p$-component (where the $s$-component is oscillating parallel to the sample surface, and the $p$-one parallel to the plane of incidence). The ratio of the reflectivity of these two components ( $r_{s}$ for the $s$-component and $r_{p}$ for the $p$-component) characterises the polarisation change and is expressed by the following equation:

$$
\frac{r_{p}}{r_{s}}=\tan \psi \cdot \mathrm{e}^{\mathrm{i} \Delta}
$$

where $\psi$ is the amplitude change and $\Delta$ the phase shift. In the thin film limit at the air/water interface (i.e., film thickness << laser wavelength), $\Delta$ is much more sensitive to changes in the amount adsorbed at the interface than $\psi$ [25]. Therefore, the time-dependent changes in phase shift $(\Delta \Delta)$ were measured, with $\Delta \Delta(t)=\Delta(t)-\Delta\left(t_{0}\right)$, where $\Delta\left(t_{0}\right)$ is the phase shift at the beginning of a given experiment, namely, the phase shift of the bare air/water interface $\left(\Delta_{0}\right)$ for BS adsorption at the air/water interface, or that of the pure DPPC monolayer ( $\left.\triangle_{\mathrm{DPPC}}\right)$ for BS interaction with this film at the air/water interface. The instrument was mounted on top of the LT to measure, simultaneously, the surface pressure and phase shift changes for the same sample. Data were acquired at a rate of $0.2 \mathrm{~Hz}$, using the Igor Pro software. Each experiment was repeated twice; a representative measurement of each is shown.

\section{Brewster angle microscopy (BAM)}

Surface pressure measurements with the LT were complemented by contemporaneous BAM measurements, which enabled the visualisation of the spatial structure of the interfacial layer. Brewster angle micrographs were obtained using a Nanofilm EP3 instrument equipped with a 532-nm-wavelength laser and a 10x objective lens (Accurion GmbH, Göttingen, Germany), and 
mounted on top of the LT. The angles of the laser and camera were identical and fixed to the value of the Brewster angle for the pure air/water interface $\left(\alpha=53.1^{\circ}\right.$ at this wavelength, with $\tan \alpha=\frac{n_{1}}{n_{2}}$, where $n_{i}$ is the refractive index of each medium at the interface). At this specific angle, the $p$-polarised light is fully transmitted from the air/water interface, thus giving a black image, while in the presence of material at the interface, the optical properties of the interface vary, thus resulting in the appearance of brighter zones due to the reflection of some $p$ polarised light. Images were captured by the built-in charge-coupled device camera, using the EP3View software, which was also employed to subtract the background.

\section{X-ray reflectometry (XRR)}

XRR experiments were performed using an Empyrean diffractometer (Malvern Panalytical S.A.S., Limeil-Brevannes, France). The instrument was configured with an incoming monochromatic X-ray beam of wavelength $\lambda=1.54 \AA$ ( $\mathrm{Cu}$ k $\alpha$ source), and the angle of incidence $\left(\vartheta_{i}\right.$, equal to that of detection) was varied between 0 and $5^{\circ}$, thus allowing values of the scattering vector perpendicular to the surface $\left(q_{z}\right.$, with $\left.q_{z}=\frac{4 \pi}{\lambda} \sin \vartheta_{i}\right)$ ranging from 0 to $0.35 \AA^{-1}$ to be obtained. Reflectivity $(R)$, which is the ratio between the reflected and incident intensities, was measured as a function of $q_{z}$ in this range.

Because X-rays are not sensitive enough to distinguish between organic molecules, XRR measurements were only carried out on the pure BS films adsorbed at the air/water interface. BS concentrations below, around, and above their critical micelle concentration (CMC) (i.e., 1, 5 and $10 \mathrm{mM}$ ) were selected because different interfacial behaviours were observed with the LT. XRR experiments were performed in ultrapure water, as the contrast with BS was high enough (Table 1).

Table 1: Calculated electron density of each component

\begin{tabular}{|ccc|}
\hline \multicolumn{2}{|c}{ Component } & Electron density $\left(.10^{-6}\right)\left(\AA^{-2}\right)$ \\
\hline \multirow{2}{*}{ Subphase } & $\mathrm{H}_{2} \mathrm{O}$ & 9.47 \\
& $\mathrm{NaTC}$ & 12.05 \\
\multirow{2}{*}{ BS } & $\mathrm{NaTDC}$ & 12.16 \\
&
\end{tabular}

XRR curves were analysed with the Aurore software [26] following two different modelling approaches: when the layer formed at the air/water interface by BS molecules was very diffuse, the evolution of the air/water interfacial roughness $(\sigma)$ was monitored and the 
thickness $(t)$ calculated as follows: $t=2.35 . \sigma$, while a model assuming a single layer characterised by a specific thickness $(t)$, electron density and $\sigma$, was used to fit a more dense, well-defined film. The result of the XRR data analysis is the electron density profile along the direction perpendicular to the surface $(z)$, which is directly related to the distribution of each molecular component in this direction.

\section{Neutron reflectometry (NR)}

NR measurements were performed on FIGARO, the ILL time-of-flight neutron reflectometer [27]. This instrument uses an incoming polychromatic neutron beam with wavelengths $(\lambda)$ ranging from 2 to $20 \AA$, with a $7 \% \mathrm{~d} \lambda / \lambda$ resolution. In order to cover a similar $q_{z}$ range as the one obtained with XRR, two different incident angles, $\theta_{1}=0.622^{\circ}$ and $\theta_{2}=3.780^{\circ}$, were employed on FIGARO; $q_{z}$ values ranging from 0.01 to $0.25 \AA^{-1}$ were thus accessible. As for XRR, $R$ was measured as a function of $q_{z}$ in this range.

The same BS concentrations as those used for XRR experiments were employed. Contrastvariation, which enables highlighting different components by simple isotopic deuteration, was performed by spreading the same phospholipid monolayer onto the surface of two subphases differing by their $\mathrm{D}_{2} \mathrm{O} / \mathrm{H}_{2} \mathrm{O}$ ratio: pure $\mathrm{D}_{2} \mathrm{O}$ and $8.1 \% \mathrm{D}_{2} \mathrm{O} / 91.9 \% \mathrm{H}_{2} \mathrm{O}$ ratio, corresponding to ACMW (air-contrast-matched water), with a scattering length density (SLD) of zero matching air. Either a non-labelled (DPPC) or deuterated $\left(d_{75}\right.$-DPPC) lipid monolayer was formed onto the clean water surface (either $\mathrm{D}_{2} \mathrm{O}$ or $\mathrm{ACMW}$ ). In this way, different parts of the system were highlighted: the DPPC/ $D_{2} \mathrm{O}$ system made both BS and lipid molecules visible, while the use of deuterated lipids ( $d_{75}$-DPPC) in $\mathrm{D}_{2} \mathrm{O}$ and ACMW highlighted, respectively, BS location and changes in lipid interfacial film thickness and organisation (Table 2).

Table 2: Calculated SLD of each component

\begin{tabular}{|ccc|}
\hline \multicolumn{2}{|c}{ Component } & SLD $\left(.10^{-6}\right)\left(\AA^{-2}\right)$ \\
\hline \multirow{2}{*}{ DPPC } & $h_{62}$-tails & -0.41 \\
& $h_{18}$-head group & 1.75 \\
\multirow{2}{*}{ d $_{75}$-DPPC } & $\mathrm{d}_{62}$-tails & 7.66 \\
& $\mathrm{~d}_{13}$-head group & 5.68 \\
\multirow{2}{*}{ Subphase } & $\mathrm{ACMW}$ & 0 \\
& $\mathrm{D}_{2} \mathrm{O}$ & 6.33 \\
\multirow{2}{*}{ BS } & $\mathrm{NaTC}$ & 0.95 \\
& $\mathrm{NaTDC}$ & 0.90 \\
\hline
\end{tabular}


Prior to analysis, NR data were converted to reflectivity curves $R\left(q_{z}\right)$ using the COSMOS software application available for the ILL reflectometers [28]. Data analysis was performed with the Aurore software [26] and a global fitting procedure was applied for compatible data sets. The modelling approach used is the same as the one reported by Campbell et al. [29]. Briefly, the thin film present at the interface was divided into two layers, each characterised by a specific $t, S L D$, amount of water $\left(f_{\text {water }}\right)$ and $\sigma$. Because of the instrumental geometry used, the first (upper) layer in the model corresponds to the lipids acyl chains, while the second (lower) one is ascribed to the head groups region in contact with the aqueous subphase. As for XRR, the result of the NR data analysis is the SLD profile along $z$.

\section{Molecular dynamics (MD) simulations}

MD simulations were carried out to investigate the molecular-scale mechanisms governing the interaction of BS with DPPC monolayers. In doing so, each simulation system contained two DPPC monolayers, each made up of 64 lipid molecules that were separated by $60 \AA$ of water. The monolayers were constructed such that the area per lipid was $42.5 \AA^{2}$, using the CHARMM-GUI membrane builder [30-32]. BS molecules and their counterions were inserted into the aqueous phase. Two different systems containing 2 and 12 BS molecules were studied for each BS (NaTC and NaTDC). Figure 2 shows a snapshot of the simulated system containing $2 \mathrm{NaTC}$ molecules between the two DPPC monolayers. The empty space in the $z$-dimension between the two monolayers was sufficiently large to prevent interactions from occuring between the two monolayers through the periodic boundary in the z-dimension.

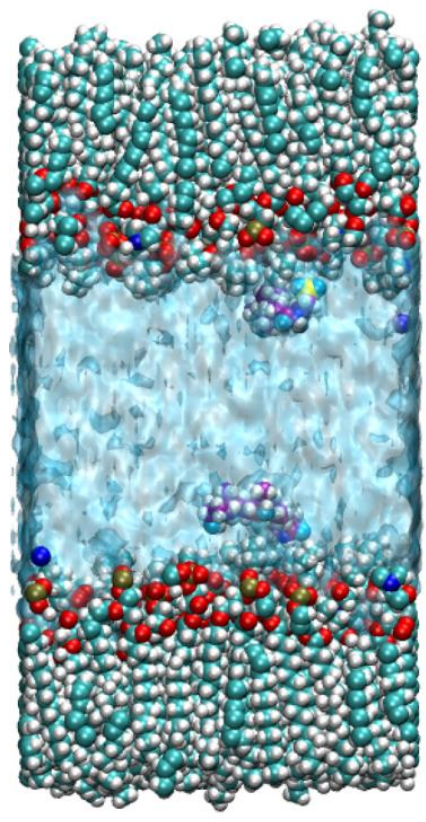


Figure 2: Snapshot of the simulated system made up of two DPPC monolayers (cyan (carbon) and red (oxygen) spheres) separated by $60 \AA$ of water (transluscent blue), which contains 2 NaTC molecules (purple spheres) and their sodium counterions.

The same simulation protocol was followed for each system. First, an energy minimisation was performed on each of the monolayer systems using 100,000 steps as the maximum number of force/energy evaluations. Then, the minimised configurations were equilibrated at $300 \mathrm{~K}$ using the NVT ensemble, in which a Langevin thermostat [33] was applied, for 400 ps. Then, a production simulation was carried out for $100 \mathrm{~ns}$ at $300 \mathrm{~K}$ using the NVT ensemble with a Nosé-Hoover thermostat [34].

All of the simulations presented in this manuscript used the LAMMPS simulation package [35]. The inter- and intra-molecular interactions of the BS, sodium counterions and DPPC molecules were described with the CHARMM force field [36-38]. The TIP3P water model [39] in its modified form, which is commonly used with the CHARMM force field [40], was used to describe the interactions involving water molecules. The van der Waals interactions were cut off at $10 \AA$ whilst the electrostatic interactions were cut off at $12 \AA$. . The PPPM method [41] was used to compute long-range Coulombic interactions. A timestep of $2 \mathrm{fs}$ was used in all simulations to ensure stable integration of Newton's equations of motion with the velocity Verlet algorithm whilst all hydrogen-containing bonds were constrained using the SHAKE algorithm [42]. 


\section{Results}

\section{BS adsorption at the air/water interface}

BS adsorption/desorption dynamics. The capacity of NaTC and NaTDC to adsorb at the air/water interface was assessed with a LT, by monitoring the evolution of the surface pressure $(\pi)$ over time after injection, into the water subphase, of either three successive amounts of BS $(1,5$ and $10 \mathrm{mM})$ (Figure $3, \mathrm{~A}, \mathrm{~B})$, or fixed BS concentrations $(0.5,5$ or $10 \mathrm{mM})$ over longer periods of time (Figure S3). These BS concentrations were selected to be below (0.5 or $1 \mathrm{mM})$, around $(5 \mathrm{mM})$, and above $(10 \mathrm{mM})$ their $\mathrm{CMC}$, which is $4-7 \mathrm{mM}$ for $\mathrm{NaTC}$ (gradual micellisation process) and $2 \mathrm{mM}$ for NaTDC (data not shown) [43].

Independently of the type of BS, the addition of a $1 \mathrm{mM}$ BS solution into the aqueous subphase leads to an instantaneous increase in surface pressure, which stabilises after a few hours. A higher surface pressure is reached for $\operatorname{NaTDC}(\pi=21 \pm 1 \mathrm{mN} / \mathrm{m}$ ) (Figure 3B), compared to $\operatorname{NaTC}(\pi=11 \pm 0.1 \mathrm{mN} / \mathrm{m}$ ) (Figure $3 \mathrm{~A}$ ). Above $5 \mathrm{mM}$, the behaviour of the two BS differs: while surface pressure does not change further with additional injections of NaTDC, which is fully micellised ( $\pi=21 \pm 2 \mathrm{mN} / \mathrm{m}$ at both 5 and $10 \mathrm{mM}$ ) (Figure 3B), the addition of $5 \mathrm{mM} \mathrm{NaTC}$ results in an increase to $\pi=21 \pm 3 \mathrm{mN} / \mathrm{m}$, which remains relatively stable at $10 \mathrm{mM}(\pi=20 \pm$ $1 \mathrm{mN} / \mathrm{m}$ ) (Figure 3A). These different patterns can be related to the higher concentration of NaTC needed to reach an aggregated state.

Similar values are obtained for both BS with individual injections of fixed concentrations (Figure S3): a surface pressure of $\pi=14 \pm 0.2 \mathrm{mN} / \mathrm{m}$ is reached at $0.5 \mathrm{mM}$, while injections of higher concentrations of BS, 5 and $10 \mathrm{mM}$, result in an increase to $\pi=23 \pm 1 \mathrm{mN} / \mathrm{m}$. These results are consistent with surface pressure values at the air/water interface reported elsewhere $[11,18,22]$. Slight differences in the kinetics of adsorption can be explained by different stirring conditions of the different set-ups.

Ellipsometry performed at the same time gives information on the amount of material adsorbed at the interface [25], by monitoring the phase shift $(\Delta \Delta)$ (Figure 3, C, D). For both BS, the phase shift increases upon injection of $1 \mathrm{mM} \mathrm{BS}$ and rapidly reaches a near plateau at $\Delta \Delta$ $=0.024 \pm 0.003^{\circ}$ and $\Delta \Delta=0.031 \pm 0.005^{\circ}$ for NaTC and NaTDC, respectively (Figure 3, C, D). At higher concentrations, again, the behaviour of the two BS diverges: while the addition of NaTC induces a further increase in phase shift (stabilising at $\Delta \Delta=0.029 \pm 0.004^{\circ}$, at $10 \mathrm{mM}$ ) 
(Figure 3C), the injection of 5 and $10 \mathrm{mM} \mathrm{NaTDC}$ leads to a gradual decrease to $\Delta \Delta=0.023 \pm$ $0.004^{\circ}$ and $\Delta \Delta=0.020 \pm 0.004^{\circ}$, respectively (Figure 3D).

NaTC
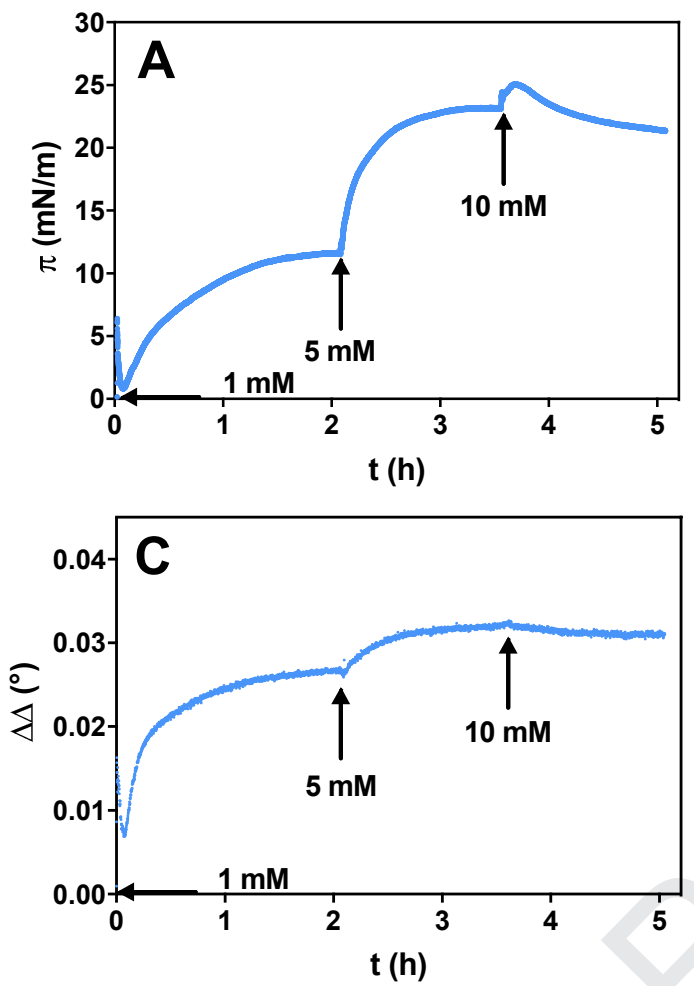

NaTDC

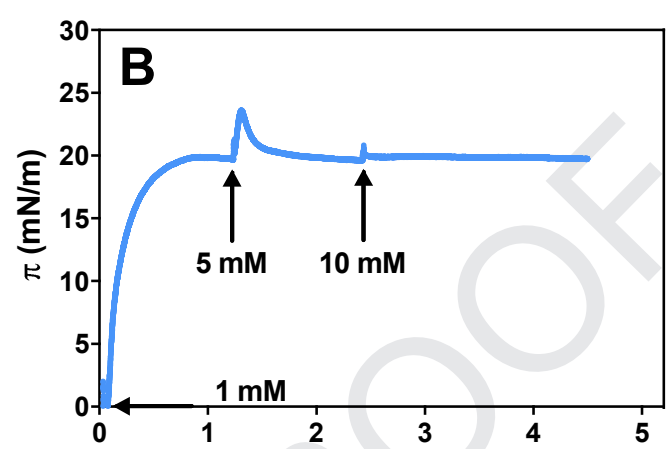

$\mathbf{t}$ (h)

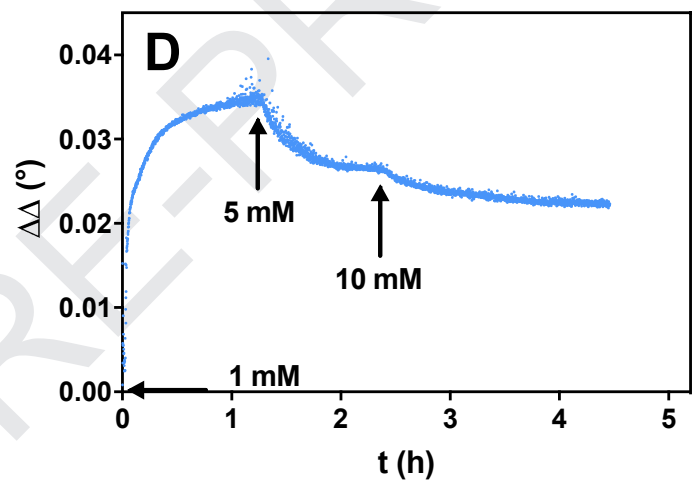

Figure 3: Time-dependent evolution of the surface pressure $(\pi)$ measured by a LT $(A, B)$, and phase shift $\left(\Delta \Delta(t)=\Delta(t)-\Delta_{0}\right)$ measured by ellipsometry (C, D), upon successive injections of either $\operatorname{NaTC}(A, C)$ or $\operatorname{NaTDC}(B, D)$ into the aqueous subphase (at $23 \pm 2^{\circ} \mathrm{C}$ ). Each addition is shown by an arrow, together with the corresponding BS concentration achieved in the subphase. Each experiment was reproduced twice, and a representative measurement was selected for each experiment.

BS interfacial film structure. A molecular-level characterisation of the BS films was obtained in the lateral (BAM, Figure 4) and perpendicular (XRR, Figures 5 and S3, Table S1) directions, with resolutions of ca. 1,000 $\mathrm{nm}$ (BAM) and $<1 \mathrm{~nm}$ (XRR). 


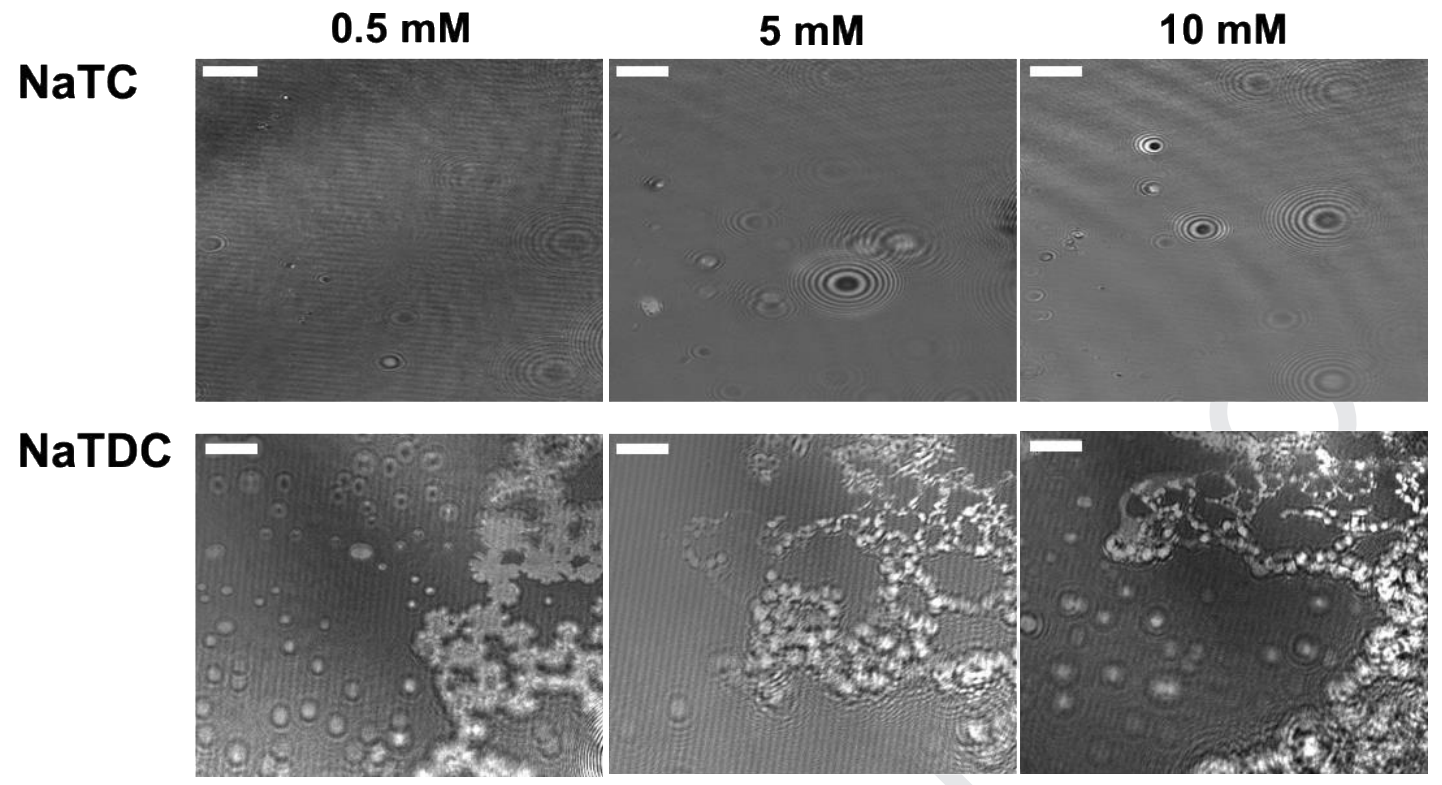

Figure 4: Evolution of BS film formation and organisation observed with a Brewster angle microscope, upon successive injections of BS into the aqueous subphase: NaTC, NaTDC (at $23 \pm 2^{\circ} \mathrm{C}$ ). The scale bar of the BAM images is $50 \mu \mathrm{m}$. Stripes are caused by stirring-induced vibrations of the water subphase and black, circular shapes are due to dust grains.

At the Brewster angle of the bare air/water interface, no light is reflected from the interface, giving a dark background. Upon successive additions of NaTC into the water subphase, the image becomes uniformly brighter, showing the formation of a homogeneous layer in the lateral direction, with a refractive index different from water (Figure 4). Instead, successive injections of NaTDC result in small, numerous brighter islands on the water surface, characterised by a specific refractive index.
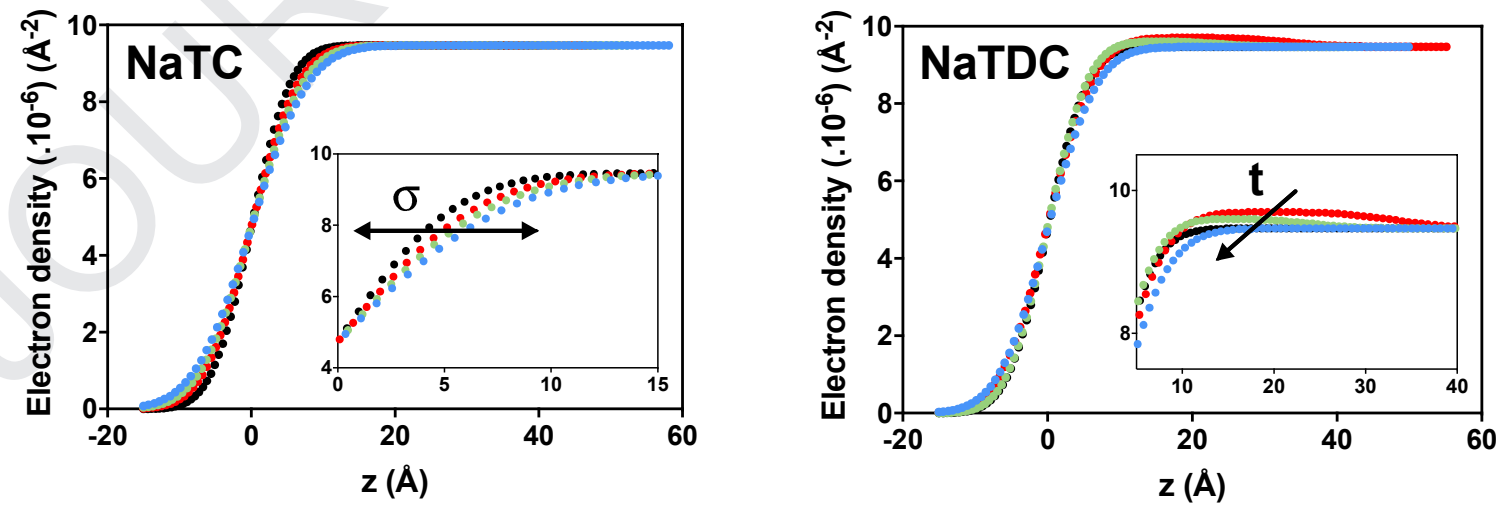

Figure 5: Evolution of the electron density profile of the interfacial film along the direction perpendicular to the surface $(z)$ obtained from XRR by successive injections of BS into the aqueous subphase: NaTC, NaTDC (at $23 \pm 2^{\circ} \mathrm{C}$ ). BS concentrations below ((प) $1 \mathrm{mM})$, around ((प) $5 \mathrm{mM})$, and above ((प) $10 \mathrm{mM}$ ) their $\mathrm{CMC}$ were selected because different interfacial behaviours were observed with the LT. The electron density profile of the bare air/water interface ( $\square$ ) is also shown. 
The successive additions of $\mathrm{NaTC}$ into the water subphase lead to the formation of a very diffuse layer, whose interfacial roughness (and thus thickness) increases with the amount of $\mathrm{BS}$ (from $\sigma=4 \AA$ (or $\mathrm{t}=9 \AA$ ) in the absence of NaTC, to $\sigma=5,6$ and $6 \AA$ (or $\mathrm{t}=12,14$ and $14 \AA$ ) in the presence of, respectively, 1, 5 and $10 \mathrm{mM}$ ) (Figures 5 and S4, Table S1). NaTDC forms a significantly thicker film at the air/water interface at $1 \mathrm{mM}(\mathrm{t}=33 \AA)$, while the addition of further NaTDC molecules results in a significant thinning of the layer ( $t=24 \AA$ at $5 \mathrm{mM}$ ); at 10 $\mathrm{mM}$, the film becomes too diffuse to be fitted using a single-layer model (at this high concentration, NaTDC forms a layer with an interfacial roughness of $\sigma=5 \AA$ (or $t=12 \AA$ ), a value very similar to those obtained for the NaTC adsorbed layer).

BS interaction with a DPPC monolayer at the air/water interface. First, the optimal lipid density, or monolayer surface pressure, leading to the most efficient adsorption of BS within the liquid-condensed phase, where the lipid monolayer is uniform and compacted $(25<\pi<$ $45 \mathrm{mN} / \mathrm{m}$ ), was determined. BS molecules were injected into the water subphase below lipid monolayers prepared at different surface pressures $\left(\pi_{\mathrm{DPPC}}=25,35,45 \mathrm{mN} / \mathrm{m}\right)$, and the least packed and ordered lipid film within the liquid-condensed phase $\left(\pi_{D P P C}=25 \mathrm{mN} / \mathrm{m}\right)$ was selected, as it showed the highest extent of BS adsorption (data not shown). Following this, three bile salts, NaTC, NaTDC and NaGDC, were injected into the water subphase below the DPPC monolayer ( $\pi_{\mathrm{DPPC}}=25 \pm 2 \mathrm{mN} / \mathrm{m}$ ), either by increasing BS concentration stepwise (10 successive injections, each spaced by one hour from the next) (Figure 6), or by adding a fixed concentration $(0.5,1,5,10$ or $20 \mathrm{mM}$ ) and monitoring over longer times ( $\mathrm{t}>2.5 \mathrm{~h}$ ) (Figure S5). The results are shown as a change in surface pressure: $\Delta \pi(t)$, with $\Delta \pi(t)=\pi(t)-\pi_{\mathrm{DPPC}}$, where $\pi_{D P P C}$ is the initial DPPC monolayer surface pressure.

The surface pressure evolution is strikingly similar for NaTDC and NaGDC (which bears a different amino acid group - glyco, instead of tauro -, but the same steroid backbone), and differs for NaTC (Figure 6). In the case of NaTC, three different regions are observed: at low concentrations $(1-2 \mathrm{mM})$, the surface pressure rises up to a plateau, at $\Delta \pi=19 \pm 2 \mathrm{mN} / \mathrm{m}$; subsequent additions of $\mathrm{BS}(3-6 \mathrm{mM})$ induce a much weaker increase, followed by a decrease above 7-8 mM. Instead, the first addition (1 mM) of either NaTDC or NaGDC induces a sharp increase in surface pressure $(\Delta \pi=27 \pm 1 \mathrm{mN} / \mathrm{m})$, which stays relatively constant around $2-3$ $\mathrm{mM}$, and then steeply drops above $4 \mathrm{mM}$. 
The interaction of NaTC and NaTDC with the DPPC film monitored over longer times (Figure S5) also shows a concentration-dependent adsorption pattern: up to a threshold concentration, which depends on the BS used (10 mM for NaTC and $1 \mathrm{mM}$ for NaTDC), the surface pressure increases to stabilise at $\Delta \pi=23 \pm 1 \mathrm{mN} / \mathrm{m}$; at higher concentrations of BS, injection into the subphase results in a small peak surface pressure increase, which then stabilises at a lower value $(\Delta \pi=11 \pm 3 \mathrm{mN} / \mathrm{m})$. The transient peaks observed with the $\mathrm{LT}$ upon injection of BS (particularly marked for NaTDC) (Figures 6, S5, and S6, A, B) were also found with ellipsometry (Figure S6, C, D), and both in the presence (Figure S6, A, C) and absence (Figure S6, B, D) of stirring; they were therefore attributed to transient adsorption/desorption processes. 

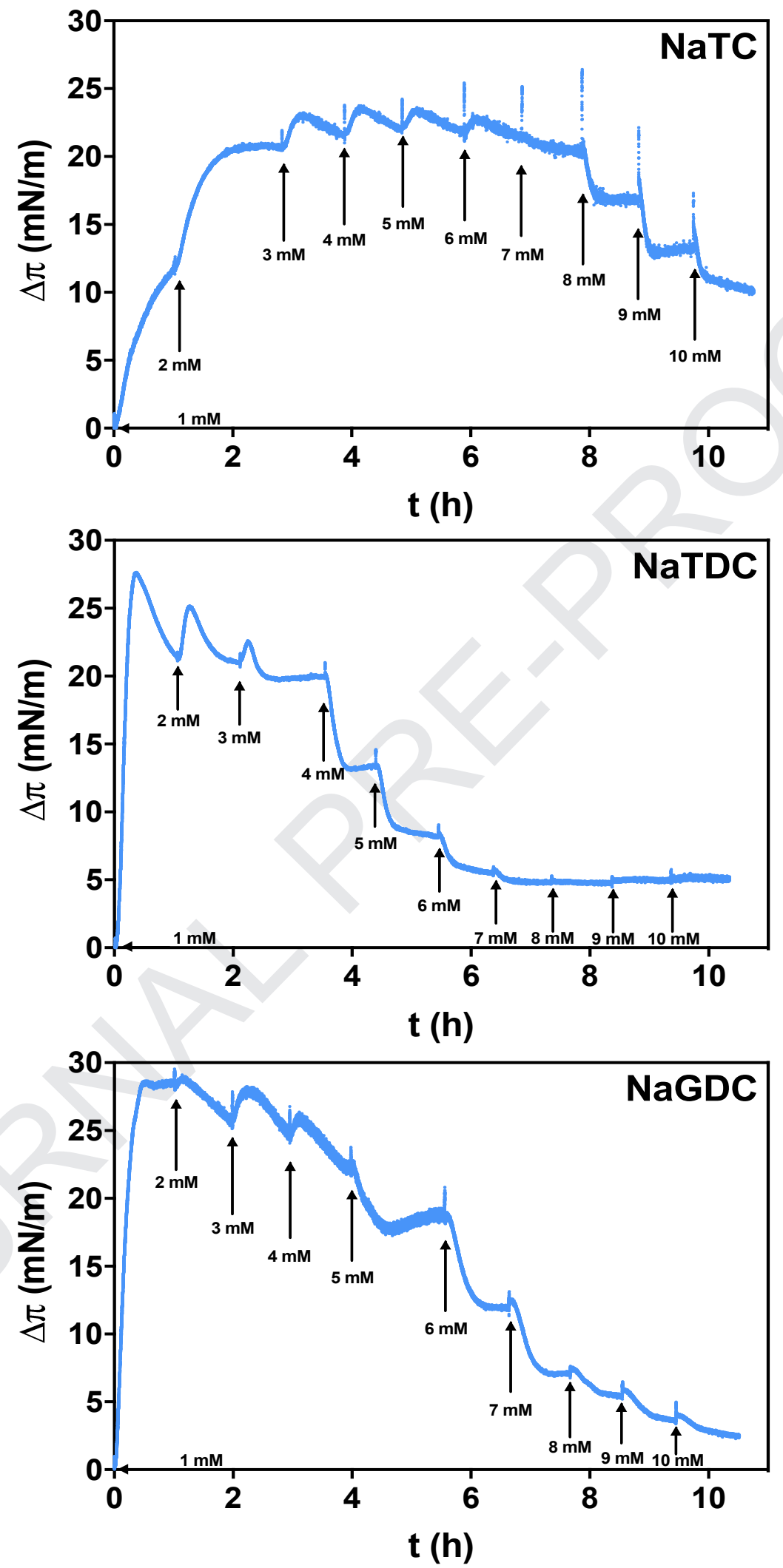

Figure 6: Time-dependent evolution of the surface pressure $\left(\Delta \pi(t)=\pi(t)-\pi_{\mathrm{DPPC}}\right)$ measured by a LT, upon successive injections of BS into the aqueous subphase: NaTC, NaTDC, NaGDC (at $23 \pm 2^{\circ} \mathrm{C}$ ). The lipids were spread onto water at $\pi_{D P P C}$ $=25 \pm 2 \mathrm{mN} / \mathrm{m}$. Each addition is shown by an arrow, together with the corresponding BS concentration achieved in the subphase. Each experiment was reproduced twice, and a representative measurement was selected for each BS. 
From the MD trajectories, the interaction of NaTC and NaTDC with an ordered DPPC monolayer was studied (Figure 7). Figure 7A shows the fraction of time that a BS molecule is bound to the monolayer, over the course of the trajectory of each simulation. The results show that, for both NaTC and NaTDC, BS molecules as unimers (i.e., below the CMC) spend more time at the lipid interface ( $30 \%$ and $40 \%$ of the time for NaTC and NaTDC, respectively) than they do when in aggregates (i.e., above the $\mathrm{CMC}$ ); in the aggregated state, they are both found to adsorb onto the mo9nolayer interface approximately $20 \%$ of the time. Figure $7 \mathrm{~B}$ shows the probability that any given NaTC or NaTDC molecule stays bound to the DPPC monolayer for a certain amount of time. NaTC molecules have nearly identical probabilities of staying bound to the interface for a given amount of time when they are isolated and aggregated. However, $\mathrm{NaTDC}$ molecules are significantly more likely to remain bound for longer times when they are as unimers than when aggregated.
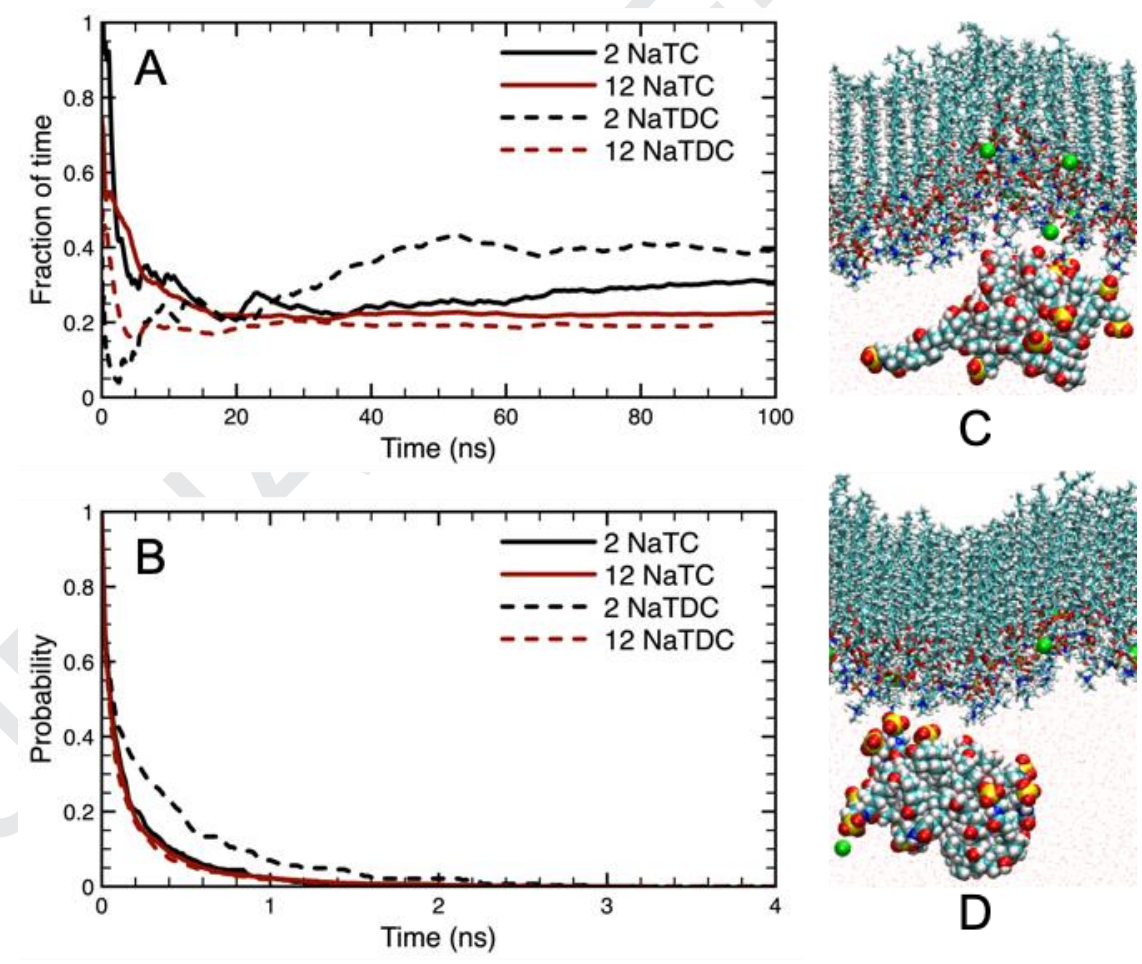

Figure 7: Binding of NaTC and NaTDC to DPPC monolayers, from MD simulations. (A) Fraction of time that NaTC (-) and NaTDC (- - -) are adsorbed to the interface of the DPPC monolayer during the course of the simulations, with 2 (black) and 12 (red) BS. (B) The survival probability of a BS molecule at the interface of the DPPC monolayer as a function of time, for $\operatorname{NaTC}(-)$ and NaTDC (- - ), in the simulations with 2 (black) and 12 (red) BS. (C, D) Snapshots of the simulations with 12 $\operatorname{NaTC}(C)$ and 12 NaTDC (D) molecules, as they interact with the DPPC monolayers.

In order to understand these differences, the pairs of atoms that are most commonly found to interact on the BS molecule and the DPPC head group moiety were measured. Figure 8 
shows the probability that a heavy atom on the BS is bound to a heavy atom in the phosphatidylcholine head group (the labels on either axis are defined in Supporting Information, Figure S7). In all cases, the most common interaction between the BS and the DPPC head group is through the sulfate group of the BS (S, O5 (04) , 06 (05), 07 (06) for NaTC (NaTDC)) and the choline head group of the lipid ( $N, \mathrm{C11}, \mathrm{C12}, \mathrm{C13}, \mathrm{C14}, \mathrm{C} 15)$. In the aggregated state (Figure 8, B, D), the oxygen at the opposite end of the BS molecule (O1) was found to commonly interact with the choline head group as well, whereas it was not nearly as common with isolated BS molecules (Figure 8, A, C).

While BS seem to most commonly interact with the DPPC choline head group, they also interact with its phosphate group. In the systems with isolated NaTC (Figure 8A) and NaTDC (Figure 8C), BS molecules were found to make contact with the phosphate group (P, O11, 012, $013,014)$ for approximately $5 \%$ of the time that they are bound. In the aggregated state, the amount of time that they bind to the phosphate group decreases for both NaTC (Figure 8B) and NaTDC (Figure 8D). However, the decrease is more significant for NaTDC ( $1 \%$ of the time), as compared to $\mathrm{NaTC}(3 \%)$.

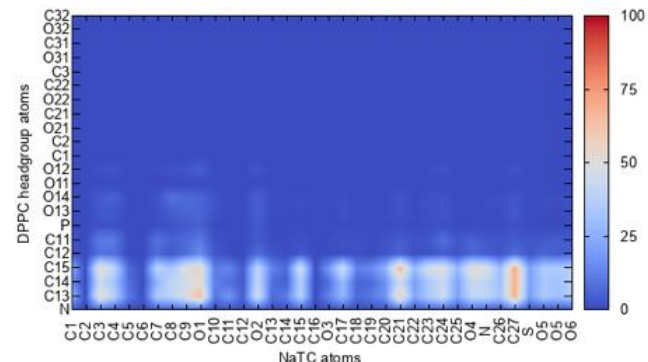

A. $2 \mathrm{NaTC}$

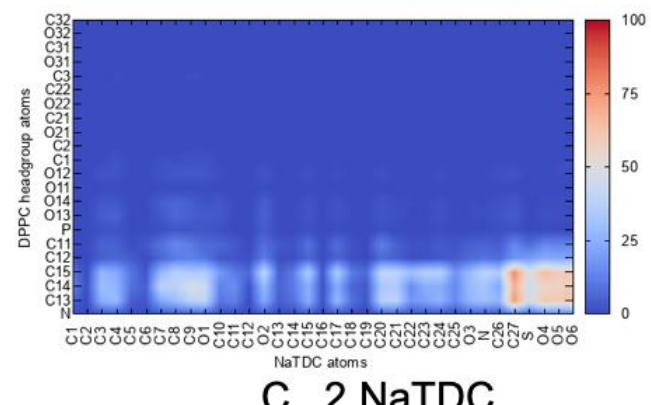

C. 2 NaTDC

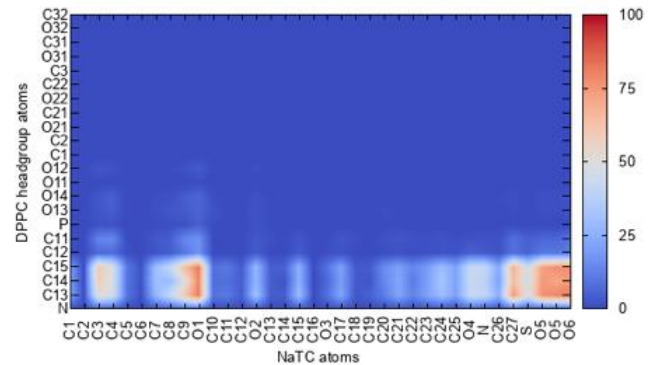

B. $12 \mathrm{NaTC}$

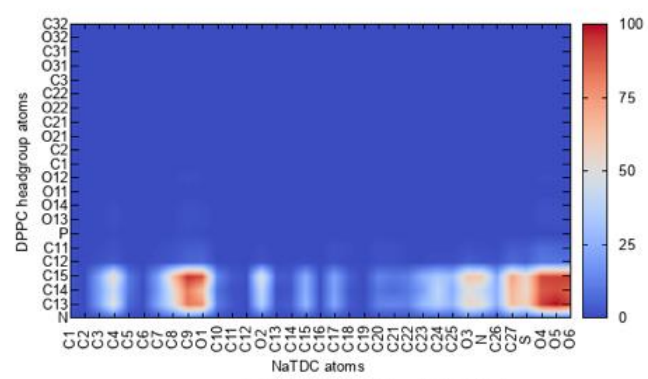

D. $12 \mathrm{NaTDC}$

Figure 8: Interaction maps for $\operatorname{NaTC}(A, B)$ and $\operatorname{NaTDC}(C, D)$ with the head group of the DPPC molecule in a monolayer. (A, C) Interactions of the two BS for the simulated systems with 2 BS molecules. (B, D) Interactions of the two BS for the simulated systems with 12 BS molecules. Note, the atom labels used on the two axis are defined in Figure S7. 
Morphology of BS/lipid films. Next, structural changes induced by the two BS on the DPPC monolayer were monitored by BAM (Figures 9, S8 and S9) and NR (Figures 10, S11 and S12, Table S2).

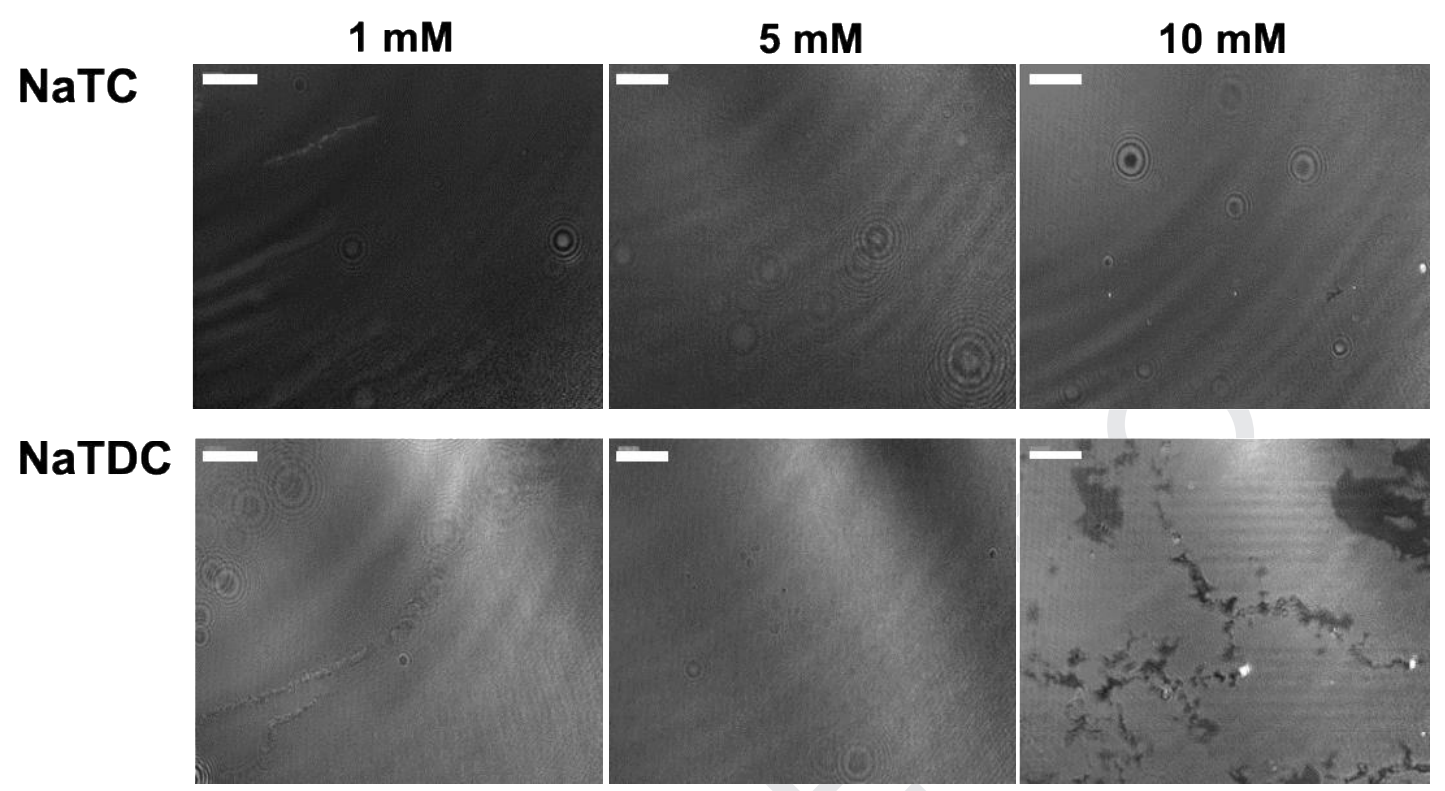

Figure 9: Evolution of the lipid monolayer organisation observed with a Brewster angle microscope, upon successive injections of BS into the aqueous subphase: $\mathrm{NaTC}, \mathrm{NaTDC}$ (at $23 \pm 2^{\circ} \mathrm{C}$ ). The lipids were spread onto water at $\pi_{\mathrm{DPPC}}=25 \pm 2$ $\mathrm{mN} / \mathrm{m}$. Three BS concentrations are shown here, and additional ones are displayed in Supporting Information (Figure S8). The scale bar of the BAM images is $\mathbf{5 0} \mu \mathrm{m}$. Stripes are caused by stirring-induced vibrations of the water subphase and (black or white) circular shapes are due to dust grains. The BAM image obtained with the pure DPPC monolayer can be found in Figure $\mathbf{S 8 .}$

BAM images show that the injection of NaTC does not affect the lipid film structure (Figures 9 and S8), while, instead, the addition of NaTDC above $9 \mathrm{mM}$ leads to a patterning of the surface, revealing the presence of different packing phases in the monolayer (Figures 9, S8 and S9).

Using NR, the internal structure of the lipid film at the sub-nanometre length scale was obtained using contrast variation, by selective deuteration of the lipids (DPPC and $d_{75}$-DPPC) and solvent $\left(\mathrm{H}_{2} \mathrm{O} / \mathrm{D}_{2} \mathrm{O}\right.$ and $\left.\mathrm{D}_{2} \mathrm{O}\right)$. We verified that DPPC (Figure S1A) and $\mathrm{d}_{75}-\mathrm{DPPC}$ (Figure S1B) exhibited a similar $\pi-A$ isotherm, and thus a similar packing state at the same surface pressure (Figure S10); measurements were consistent with literature values for DPPC [44] and $\mathrm{d}_{75}$-DPPC [45]. 

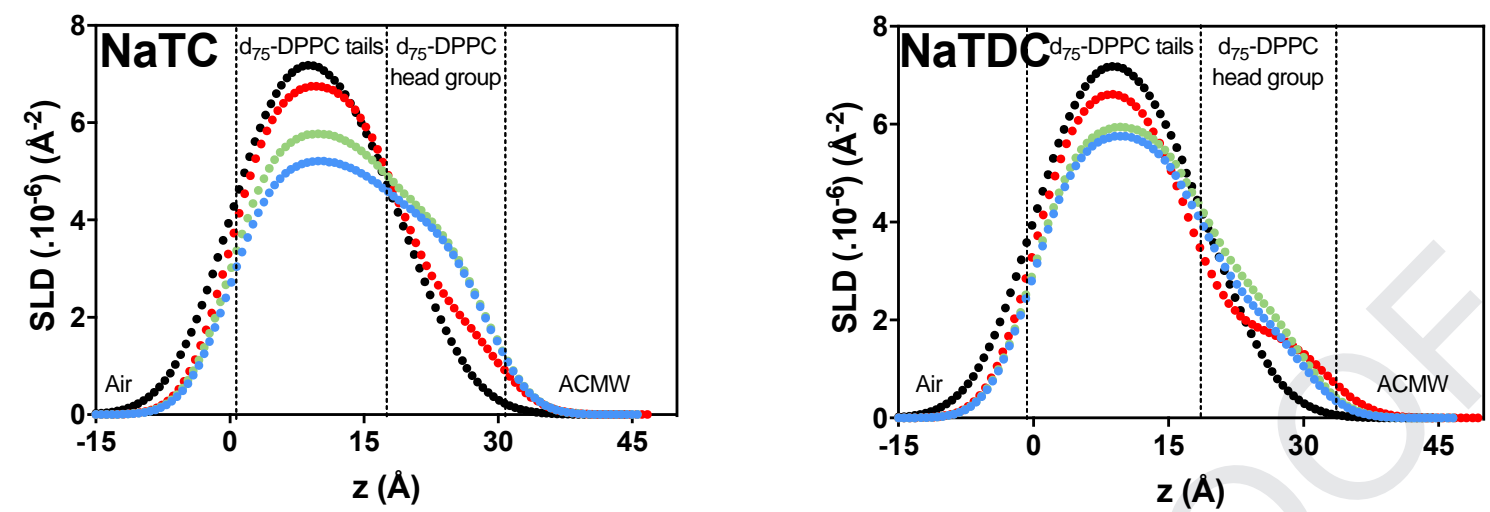

Figure 10: Evolution of the scattering length density $(S L D)$ profile of the interfacial film along the direction perpendicular to the surface $(z)$ obtained by successive injections of BS into the aqueous subphase: NaTC, NaTDC (at $23 \pm 2^{\circ} \mathrm{C}$ ). The lipids were spread onto water at $\pi_{\mathrm{DPPC}}=25 \pm 2 \mathrm{mN} / \mathrm{m}$, thus forming a pure monolayer ( $\square$ ). BS concentrations below (( $\left.\left.\square\right) 1 \mathrm{mM}\right)$, around ((प) $5 \mathrm{mM})$, and above $((\mathrm{D}) 10 \mathrm{mM}$ ) their $\mathrm{CMC}$ were selected because different interfacial behaviours were observed with the LT. These SLD profiles were recorded in ACMW (SLD of 0 ), on which a $d_{75}$-DPPC monolayer (SLD of 7.66.10-6 $\AA^{-2}$ for the tails and 5.68.10-6 $\AA^{-2}$ for the head group) was prepared; NaTC has a SLD of 0.95.10-6 $\AA^{-2}$ and NaTDC of $0.90 .10^{-6} \AA^{-2}$. The $S L D$ profiles obtained in the other conditions of contrast are displayed in Supporting Information (Figure S12).

The proportions of lipid, water and BS in the interfacial film were modelled by assuming that only air could penetrate the tails, while both water and BS could go into the head group region, but not into the tails (further detail on the fitting process can be found in Supporting Information). Based on these assumptions, the head group region of the pure lipid monolayer (in the absence of BS) was found to comprise of 90\% lipids and 10\% water (Figures 10, S11 and S12, Table S2). For both BS, successive additions of either NaTC or NaTDC under this lipid film lead to a gradual decrease in the SLD of the tails layer, thus indicating a transition from a condensed, uniform phospholipid film to a less compacted and more disorganised one. This observation is in agreement with results from atomic force microscopy, using DPPC and a mixture of NaTC and NaGDC [20]. In concurrence with the results obtained with all the other techniques, the injection of the two BS into the water subphase generates different structures at the lipid/water interface. With NaTC, two concentration regimes can be distinguished: at low concentration (1 mM), BS molecules adsorb significantly at the interface, reaching a 1:1 BS/DPPC molar ratio, while at higher concentrations, the BS/DPPC molar ratio decreases sharply (to $0.1: 1$, at $5 \mathrm{mM}$ ), until there is no $\mathrm{BS}$ at the lipid/water interface at $10 \mathrm{mM}$. In addition, at this very high BS concentration $(10 \mathrm{mM})$, the proportion of DPPC at the air/water 
interface decreases to $70 \%$, showing a less compacted film. In contrast, the injection of NaTDC results in a net decrease in the amount of DPPC at the interface, from $90 \%$ (without BS) to approximately $40 \%$ (with BS), independently of the BS concentration. Moreover, in contrast to NaTC, a BS/DPPC molar ratio of 0:1 was obtained for NaTDC at all BS concentrations, indicating that NaTDC molecules do not penetrate the head group layer. 


\section{Discussion}

The aim of this work was to study and compare the interfacial properties of two BS to understand the impact of their molecular structure on the specific roles they play in lipolysis. For this purpose, their adsorption at an air/water interface and their interaction with a phospholipid monolayer - mimicking the physiological components present at the interface of fat droplets - were characterised. The two BS, differing in the structure of their bile acid moiety (NaTC has an additional hydroxyl group (Figure 1)), were selected based on previously reported distinct adsorption/desorption dynamics on hydrophobic surfaces [19].

First, BS adsorption properties at the air/water interface were evaluated. Surface pressure measurements show that the injection of BS results in an instantaneous increase in surface pressure, independently of the type of BS, reflecting a very fast adsorption process (Figures 3, A, B, and S3). These rapid dynamics have been observed previously $[11,18,22]$, and are attributed to the planar structure and large surface area of these molecules [46-48]. This unusual flat configuration also explains the relatively low surface pressure $(\pi=21-23 \mathrm{mN} / \mathrm{m})$ obtained after equilibration (Figures 3, A, B, and S3). In comparison, traditional surfactants typically reach values around $\pi=40-50 \mathrm{mN} / \mathrm{m}$. Ellipsometry (Figure 3, C, D) and XRR (Figure 5, Table S1) measurements reveal different adsorption/desorption behaviour for the two BS. Successive injections of NaTC result in an increase in surface pressure (Figure $3 \mathrm{~A}$ ), phase shift (Figure $3 C$ ) and interfacial roughness (Figure 5, Table S1), which all correlate with a higher amount of material adsorbed at the interface [25]. The combination of the three techniques (LT, ellipsometry and XRR) thus confirms that NaTC adsorbs at the interface, until it forms a stable film. In addition, BAM (Figure 4) and XRR (Figure 5, Table S1) measurements indicate that the layers formed by NaTC are laterally homogeneous, but diffuse in the perpendicular direction. In contrast, successive additions of NaTDC induce a decrease in phase shift above 5 $\mathrm{mM}$ (Figure 3D), in other words, the desorption of material, while the surface pressure remains constant (Figure 3B). In conjunction with the ellipsometry measurements, the characterisation of NaTDC adsorbed layer by XRR (Figure 5, Table S1) shows that, at low concentrations (1 mM), NaTDC adsorbs readily at the air/water interface, forming a relatively thick film, while the addition of further BS ( $5 \mathrm{mM}$ ) leads to a decrease in layer thickness, until a very diffuse film is formed (at $10 \mathrm{mM}$ ), thus demonstrating that NaTDC partially leaves the interface as its concentration in the bulk increases. The discrepancy between the stabilisation 
of the surface pressure, which suggests a stabilisation of the film, and the decrease in both phase shift and film thickness, which implies the desorption of material, can be explained by the formation of buried layers beneath the monolayer at the air/water interface. Any event occurring in these sublayers (either adsorption or desorption) would not affect surface pressure [21,49-51]. Moreover, unlike NaTC, which produces a laterally uniform film at the air/water interface, the adsorption of NaTDC leads to irregular patterns in BAM images, reflecting the formation of islands on the water surface (Figure 4). Based on these results, it is clear that the small structural difference between the two BS affects their adsorption behaviour at the air/water interface, with NaTC being more prone to remain at the interface, despite its lower hydrophobicity [52].

In the second part, the interaction of BS with a DPPC monolayer was studied, mimicking the interfacial processes taking place when a hydrosoluble BS molecule approaches a fat droplet.

Successive injections of NaTC $(1-6 \mathrm{mM})$ below this monolayer induce an increase in surface pressure (Figure 6), suggesting adsorption of the BS. This observation is confirmed by NR, which shows that NaTC adsorbs into the lipid head group region, at a 1:1 BS/DPPC molar ratio at $1 \mathrm{mM}$ (Figures 10 and S12, Table S2). MD simulations indicate that NaTC primarily interacts with the choline head group of DPPC, and does occasionally penetrate deeper into the lipid membrane, to the level of the phosphate group (Figure 8, A, B and S6, A, C). Above ca. $7 \mathrm{mM}$, however, a decrease in surface pressure is measured, which could suggest the desorption of NaTC from the interface or, alternatively, the displacement of DPPC molecules into the subphase, through their incorporation into mixed micelles (Figure 6). BAM images show that the interfacial layer remains homogeneous, suggesting the absence of any lateral disorganisation (which could occur by the removal of DPPC molecules) (Figures 9 and S8). NR data at these concentrations show a transition to a less compacted and more disordered DPPC film, as well as a decrease in the amount of NaTC in the lipid layer (from 1:1 BS/DPPC at $1 \mathrm{mM}$ to $0.1: 1$ at $5 \mathrm{mM}$ and 0:1 at $10 \mathrm{mM}$ ) (Figures 10 and S12, Table S2). The apparent discrepancy between BAM and NR results can be explained by the rather low lateral resolution of the Brewster angle microscope. Overall, these results clearly imply that, at high concentrations, $\mathrm{NaTC}$ desorbs from the interface and removes some DPPC molecules in the process. This change in behaviour (from adsorption to desorption) corresponds to the onset of micellisation (4- $7 \mathrm{mM}$ for $\mathrm{NaTC}$ ). The results of MD simulations corroborate these observations, showing 
a higher probability of NaTC molecules to interact with the lipid film in their non-aggregated state, compared to their micellised state (Figure 7).

In contrast to NaTC, the addition of NaTDC leads to a decrease in surface pressure at quite low concentrations (ca. $3 \mathrm{mM}$ ), which could be due to either BS molecules desorbing from the interface or DPPC molecules being displaced by BS (Figure 6). This behaviour suggests a lower affinity for the lipid/water interface. A similar surface pressure trend is exhibited by both NaTDC and NaGDC, stressing the importance of the steroid backbone in dictating behaviour, rather than the conjugated amino acid. NR measurements reveal that NaTDC molecules, unlike NaTC, do not penetrate deeply into the DPPC head group layer, a key difference between the two BS (Figures 10 and S12, Table S2). NaTDC induces, instead, a diffuse layer beneath the DPPC head group, at the head group/water interface. MD simulations show a drop in interaction with the monolayer upon aggregation of BS (Figure 7), which, for NaTDC, occurs at very low concentrations (2 mM). NaTDC interacts with the choline head group of DPPC, and is generally oriented such that the length of the molecule is parallel to the monolayer interface (Figure 8, C, D, and S7, B, C). As for NaTC, the addition of increasing amounts of NaTDC loosens the packing of the DPPC monolayer (Figures 10 and S12, Table S2). This disordering of the lipid monolayer is also detected in MD-simulations when BS are in the aggregated state (Figures $S 12, B, D$, and $S 13, B, D$ ), but not in the unimer state (Figures $S 12, A$, $C$, and $S 13, A, C)$. The major difference between the two BS is the strong desorption of DPPC molecules from the interface induced by NaTDC (to approximately 40\%, at all concentrations), resulting in the formation of fluid domains (Figures 10 and S12, Table S2). The addition of NaTDC was seen to induce the formation of domains with distinct organisations (i.e., lipid packing states), clearly detected by BAM above $9 \mathrm{mM}$ (Figures 9 , S8 and S9). The lack of domains at lower concentrations may be because these domains are, in size, smaller than the lateral resolution of the Brewster angle microscope. These observations taken together suggest, for NaTDC, a lipid-solubilising effect, leading to the formation of mixed micelles in the bulk.

Overall, the combination of all these experiments clearly suggest different roles during lipolysis for the two BS studied: cholate-based BS (NaTC) have a higher affinity for the interface and may thus be more prone to adsorb at the lipid/water interface, thus facilitating lipase and co-lipase adsorption, while deoxycholate derivatives (NaTDC and NaGDC) have a higher propensity to desorb and are thus more likely to be involved in removing insoluble hydrolysis 
products from the interface, by solubilising them into mixed micelles. These findings thus confirm that a very small difference in BS structure on the steroid backbone has a major impact on their adsorption/desorption behaviour and may be a key to the significance - often overlooked - of BS structural diversity. 


\section{Conclusion}

In this work, we compared the adsorption dynamics and film morphology of two BS, NaTC and NaTDC, at the air/water interface and under a phospholipid monolayer, in order to shed light on their specific roles in lipid digestion.

Both BS injected under a DPPC monolayer were found to disrupt lipid packing. However, NaTC penetrated more deeply into the lipid head group region, compared to NaTDC. In addition, NaTC remained adsorbed at the interface over a wider concentration range. Instead, NaTDC was shown to displace DPPC molecules through dynamic exchange. These findings support our hypothesis that NaTC may be more efficient at facilitating the adsorption and activity of co-lipase-dependent pancreatic lipase, while NaTDC may be more prone to remove insoluble lipolysis products from the interface, through their incorporation into mixed micelles. This hypothesis will need to be further explored in future work by enzymatic absorption studies.

The contrasting interfacial behaviour displayed by the two BS was also found to correlate with their bulk aggregation processes: the adsorption of NaTC at interfaces over a wider range of concentrations correlates with a later onset of micellisation (4-7 mM), while desorption of NaTDC occurring at low concentrations correlates with a lower CMC value (ca. $2 \mathrm{mM}$ ). Therefore, BS adsorption/desorption processes at the interface are linked to processes taking place in the bulk, which, in turn, are dictated by the chemical structure.

To our knowledge, this is the first time that the interaction between BS and a lipid monolayer - mimicking the phospholipids molecules present on the surface of a fat droplet has been characterised at the nanometre scale. Our NR studies and MD simulations establish a molecular-level description of the BS and lipid films, which is a key-step towards a better understanding of the lipolysis process and addressing the current challenges of excessive fat uptake and associated health conditions. This work will be complemented by additional NR work on lipid bilayers and at the oil/water interface (in preparation), and with bulk studies on mixed micelles of BS and the products of lipolysis, by small-angle neutron scattering and MD simulations, as well as in vitro lipolysis experiments. 


\section{Acknowledgements}

The authors acknowledge the ILL for the provision of a PhD studentship (OP) and award of beam time on FIGARO (doi: 10.5291/ILL-DATA.9-10-1488). The Partnership for Soft Condensed Matter (PSCM) is acknowledged for access to sample preparation facilities and the use of the LT, ellipsometer, Brewster angle microscope and X-ray reflectometer. OP thanks Kelly Vandera for her kind help with LT experiments, as well as Pierre Lloria and Andrea Tummino for the support provided during the acquisition of BAM images and ellipsometry measurements. Arthur Bernardeau was involved in preliminary work on MD simulations during a summer internship. PJW and MM-LG gratefully acknowledge the support of the Biotechnology and Biological Sciences Research Council (BBSRC) through the BBSRC Institute Strategic Programme Food Innovation and Health BB/R012512/1 and its constituent project BBS/E/F/000PR10345.

\section{Declarations of interest}

None 


\section{References}

[1] World Health Organization, Obesity and Overweight, (2018). http://www.who.int/mediacentre/factsheets/fs311/en/ (accessed August 21, 2018).

[2] World Health Organization, Food \& Agriculture Organization, Diet, Nutrition and the Prevention of Chronic Diseases, Geneva, 2003. http://apps.who.int/iris/bitstream/10665/42665/1/WHO_TRS_916.pdf?ua=1.

[3] J. Mei, A. Lindqvist, L. Krabisch, J.F. Rehfeld, C. Erlanson-Albertsson, Appetite suppression through delayed fat digestion, Physiol. Behav. 89 (2006) 563-568. doi:10.1016/j.physbeh.2006.07.020.

[4] M. Golding, T.J. Wooster, The influence of emulsion structure and stability on lipid digestion, Curr. Opin. Colloid Interface Sci. 15 (2010) 90-101. doi:10.1016/j.cocis.2009.11.006.

[5] P.J. Wilde, B.S. Chu, Interfacial \& colloidal aspects of lipid digestion, Adv. Colloid Interface Sci. 165 (2011) 14-22. doi:10.1016/j.cis.2011.02.004.

[6] M. Armand, Lipases and lipolysis in the human digestive tract: where do we stand?, Curr. Opin. Clin. Nutr. Metab. Care. 10 (2007) 156-164. doi:10.1097/MCO.0b013e3280177687.

[7] P. Reis, K. Holmberg, H. Watzke, M.E. Leser, R. Miller, Lipases at interfaces: a review, Adv. Colloid Interface Sci. 147-148 (2009) 237-250. doi:10.1016/j.cis.2008.06.001.

[8] J.S. Patton, M.C. Carey, Watching fat digestion, Science (80-. ). 204 (1979) 145-148. doi:10.1126/science.432636.

[9] Y. Pafumi, D. Lairon, P. Lechene de la Porte, C. Juhel, J. Storch, M. Hamosh, M. Armand, Mechanisms of inhibition of triacylglycerol hydrolysis by human gastric lipase, J. Biol. Chem. 277 (2002) 28070-28079. doi:10.1074/jbc.M202839200.

[10] A.F. Hofmann, K.J. Mysels, Bile salts as biological surfactants, Colloids and Surfaces. 30 (1987) 145-173. doi:10.1016/0166-6622(87)80207-X.

[11] J. Maldonado-Valderrama, P. Wilde, A. Macierzanka, A. Mackie, The role of bile salts in digestion, Adv. Colloid Interface Sci. 165 (2011) 36-46. doi:10.1016/j.cis.2010.12.002.

[12] L. Galantini, M.C. di Gregorio, M. Gubitosi, L. Travaglini, J. Vázquez Tato, A. Jover, F. Meijide, V.H. Soto Tellini, N. V. Pavel, Bile salts and derivatives: rigid unconventional 
amphiphiles as dispersants, carriers and superstructure building blocks, Curr. Opin. Colloid Interface Sci. 20 (2015) 170-182. doi:10.1016/j.cocis.2015.08.004.

[13] D. Madenci, S.U. Egelhaaf, Self-assembly in aqueous bile salt solutions, Curr. Opin. Colloid Interface Sci. 15 (2010) 109-115. doi:10.1016/j.cocis.2009.11.010.

[14] B. Borgström, C. Erlanson-Albertsson, T. Wieloch, Pancreatic colipase: chemistry and physiology., J. Lipid Res. 20 (1979) 805-816. http://www.ncbi.nlm.nih.gov/pubmed/385801.

[15] C. Erlanson-Albertsson, The interaction between pancreatic lipase and colipase: a protein-protein interaction regulated by a lipid, FEBS Lett. 162 (1983) 225-229. doi:10.1016/0014-5793(83)80760-1.

[16] A. Bourbon Freie, F. Ferrato, F. Carrière, M.E. Lowe, Val-407 and Ile-408 in the $\beta 5^{\prime}-$ loop of pancreatic lipase mediate lipase-colipase interactions in the presence of bile salt micelles, J. Biol. Chem. 281 (2006) 7793-7800. doi:10.1074/jbc.M512984200.

[17] S. Labourdenne, O. Brass, M. Ivanova, A. Cagna, R. Verger, Effects of colipase and bile salts on the catalytic activity of human pancreatic lipase. A study using the oil drop tensiometer, Biochemistry. 36 (1997) 3423-3429. doi:10.1021/bi961331k.

[18] J. Maldonado-Valderrama, J.L. Muros-Cobos, J.A. Holgado-Terriza, M.A. CabrerizoVílchez, Bile salts at the air-water interface: adsorption and desorption, Colloids Surfaces B Biointerfaces. 120 (2014) 176-183. doi:10.1016/j.colsurfb.2014.05.014.

[19] R. Parker, N.M. Rigby, M.J. Ridout, A.P. Gunning, P.J. Wilde, The adsorptiondesorption behaviour and structure function relationships of bile salts, Soft Matter. 10 (2014) 6457-6466. doi:10.1039/c4sm01093k.

[20] B.-S. Chu, A.P. Gunning, G.T. Rich, M.J. Ridout, R.M. Faulks, M.S.J. Wickham, V.J. Morris, P.J. Wilde, Adsorption of bile salts and pancreatic colipase and lipase onto digalactosyldiacylglycerol and dipalmitoylphosphatidylcholine monolayers, Langmuir. 26 (2010) 9782-9793. doi:10.1021/la1000446.

[21] A. Bénarouche, L. Sams, C. Bourlieu, V. Vié, V. Point, J.F. Cavalier, F. Carrière, Studying gastric lipase adsorption onto phospholipid monolayers by surface tensiometry, ellipsometry, and atomic force microscopy, in: M.H. Gelb (Ed.), Methods Enzymol. Enzymol. Membr. Interface Interfacial Enzymol. Protein-Membrane Bind., 1st ed., Elsevier Inc., San Diego, 2017: pp. 255-278. doi:10.1016/bs.mie.2016.09.039.

[22] J. Maldonado-Valderrama, N.C. Woodward, A.P. Gunning, M.J. Ridout, F.A. Husband, 
A.R. Mackie, V.J. Morris, P.J. Wilde, Interfacial characterization of $\beta$-lactoglobulin networks: displacement by bile salts, Langmuir. 24 (2008) 6759-6767. doi:10.1021/la800551u.

[23] H. Singh, A. Ye, D. Horne, Structuring food emulsions in the gastrointestinal tract to modify lipid digestion, Prog. Lipid Res. 48 (2009) 92-100.

doi:10.1016/j.plipres.2008.12.001.

[24] D.G. Dalgleish, Food emulsions - their structures and structure-forming properties, Food Hydrocoll. 20 (2006) 415-422. doi:10.1016/j.foodhyd.2005.10.009.

[25] H. Motschmann, R. Teppner, Ellipsometry in interface science, in: D. Möbius, R. Miller (Eds.), Stud. Interface Sci. - Nov. Methods to Study Interfacial Layers, 1st ed., Elsevier Science B. V., Amsterdam, 2001: pp. 1-42.

[26] Y. Gerelli, Aurore: new software for neutron reflectivity data analysis, J. Appl. Crystallogr. 49 (2016) 330-339. doi:10.1107/S1600576716000108.

[27] R.A. Campbell, H.P. Wacklin, I. Sutton, R. Cubitt, G. Fragneto, FIGARO: the new horizontal neutron reflectometer at the ILL, Eur. Phys. J. Plus. 126 (2011) 1-22. doi:10.1140/epjp/i2011-11107-8.

[28] Institut Laue-Langevin, The COSMOS software application available for the ILL reflectometers, (2018). https://www.ill.eu/fr/users-en/instruments/instrumentslist/d17/more/cosmos/ (accessed August 23, 2018).

[29] R.A. Campbell, Y. Saaka, Y. Shao, Y. Gerelli, R. Cubitt, E. Nazaruk, D. Matyszewska, M.J. Lawrence, Structure of surfactant and phospholipid monolayers at the air/water interface modeled from neutron reflectivity data, J. Colloid Interface Sci. 531 (2018) 98-108. doi:10.1016/j.jcis.2018.07.022.

[30] S. Jo, T. Kim, V.G. Iyer, W. Im, CHARMM-GUI: a web-based graphical user interface for CHARMM, J. Comput. Chem. 29 (2008) 1859-1865. doi:10.1002/jcc.20945.

[31] E.L. Wu, X. Cheng, S. Jo, H. Rui, K.C. Song, E.M. Dávila-Contreras, Y. Qi, J. Lee, V. Monje-Galvan, R.M. Venable, J.B. Klauda, W. Im, CHARMM-GUI Membrane Builder toward realistic biological membrane simulations, J. Comput. Chem. 35 (2014) 19972004. doi:10.1002/jcc.23702.

[32] J. Lee, X. Cheng, J.M. Swails, M.S. Yeom, P.K. Eastman, J.A. Lemkul, S. Wei, J. Buckner, J.C. Jeong, Y. Qi, S. Jo, V.S. Pande, D.A. Case, C.L. Brooks III, A.D. MacKerell Jr., J.B. Klauda, W. Im, CHARMM-GUI input generator for NAMD, GROMACS, AMBER, 
OpenMM, and CHARMM/OpenMM simulations using the CHARMM36 additive force field, J. Chem. Theory Comput. 12 (2016) 405-413. doi:10.1021/acs.jctc.5b00935.

[33] T. Schneider, E. Stoll, Molecular-dynamics study of a three-dimensional onecomponent model for distortive phase transitions, Phys. Rev. B. 17 (1978) 1302-1322. doi:10.1103/PhysRevB.17.1302.

[34] W.G. Hoover, Canonical dynamics: equilibrium phase-space distributions, Phys. Rev. A. 31 (1985) 1695-1697. doi:10.1103/PhysRevA.31.1695.

[35] S. Plimpton, Fast parallel algorithms for short-range molecular dynamics, J. Comput. Phys. 117 (1995) 1-19. doi:10.1006/jcph.1995.1039.

[36] K. Vanommeslaeghe, E. Hatcher, C. Acharya, S. Kundu, S. Zhong, J. Shim, E. Darian, O. Guvench, P. Lopes, I. Vorobyov, A.D. MacKerell, Jr., CHARMM general force field: a force field for drug-like molecules compatible with the CHARMM all-atom additive biological force fields, J. Comput. Chem. 31 (2010) 671-690. doi:10.1002/jcc.21367.

[37] J.B. Klauda, R.M. Venable, J.A. Freites, J.W. O'Connor, D.J. Tobias, C. MondragonRamirez, I. Vorobyov, A.D. MacKerell, Jr., R.W. Pastor, Update of the CHARMM allatom additive force field for lipids: validation on six lipid types, J. Phys. Chem. B. 114 (2010) 7830-7843. doi:10.1021/jp101759q.

[38] R.W. Pastor, A.D. MacKerell, Jr., Development of the CHARMM force field for lipids, J. Phys. Chem. Lett. 2 (2011) 1526-1532. doi:10.1021/jz200167q.

[39] W.L. Jorgensen, J. Chandrasekhar, J.D. Madura, R.W. Impey, M.L. Klein, Comparison of simple potential functions for simulating liquid water, J. Chem. Phys. 79 (1983) 926935. doi:10.1063/1.445869.

[40] W.E. Reiher, Theoretical studies of hydrogen bonding, Harvard University, 1985.

[41] T. Darden, D. York, L. Pedersen, Particle mesh Ewald: an $N \cdot \log (\mathrm{N})$ method for Ewald sums in large systems, J. Chem. Phys. 98 (1993) 10089-10092. doi:10.1063/1.464397.

[42] J.-P. Ryckaert, G. Ciccotti, H.J.. Berendsen, Numerical integration of the cartesian equations of motion of a system with constraints: molecular dynamics of $n$-alkanes, J. Comput. Phys. 23 (1977) 327-341. doi:10.1016/0021-9991(77)90098-5.

[43] K. Matsuoka, M. Maeda, Y. Moroi, Micelle formation of sodium glyco- and taurocholates and sodium glyco- and taurodeoxycholates and solubilization of cholesterol into their micelles, Colloids Surfaces B Biointerfaces. 32 (2003) 87-95. doi:10.1016/S0927-7765(03)00148-6. 
[44] K. Sabatini, J.-P. Mattila, P.K.J. Kinnunen, Interfacial behavior of cholesterol, ergosterol, and lanosterol in mixtures with DPPC and DMPC, Biophys. J. 95 (2008) 2340-2355. doi:10.1529/biophysj.108.132076.

[45] V. Rondelli, G. Fragneto, S. Motta, E. Del Favero, L. Cantù, Reflectivity from floating bilayers: can we keep the structural asymmetry?, J. Phys. Conf. Ser. 340 (2012) 1-10. doi:10.1088/1742-6596/340/1/012083.

[46] A. Tiss, S. Ransac, H. Lengsfeld, P. Hadvàry, A. Cagna, R. Verger, Surface behaviour of bile salts and tetrahydrolipstatin at air/water and oil/water interfaces, Chem. Phys. Lipids. 111 (2001) 73-85. doi:10.1016/S0009-3084(01)00149-9.

[47] N. Matubayasi, M. Kanzaki, S. Sugiyama, A. Matuzawa, Thermodynamic study of gaseous adsorbed films of sodium taurocholate at the air/water interface, Langmuir. 12 (1996) 1860-1862. doi:10.1021/la950832o.

[48] N. Matubayasi, S. Sugiyama, M. Kanzaki, A. Matuzawa, Thermodynamic studies of the adsorbed films and micelles of sodium taurodeoxycholate, J. Colloid Interface Sci. 196 (1997) 123-127. doi:10.1006/jcis.1997.5213.

[49] D.E. Graham, M.C. Phillips, Proteins at liquid interfaces. III. Molecular structures of adsorbed films, J. Colloid Interface Sci. 70 (1979) 427-439. doi:10.1016/00219797(79)90050-X.

[50] V.M. Bolanos-Garcia, A. Renault, S. Beaufils, Surface rheology and adsorption kinetics reveal the relative amphiphilicity, interfacial activity, and stability of human exchangeable apolipoproteins, Biophys. J. 94 (2008) 1735-1745. doi:10.1529/biophysj.107.115220.

[51] D.J.F. Taylor, R.K. Thomas, J.D. Hines, K. Humphreys, J. Penfold, The adsorption of oppositely charged polyelectrolyte/surfactant mixtures at the air/water interface: neutron reflection from dodecyl trimethylammonium bromide/sodium poly(styrene sulfonate) and sodium dodecyl sulfate/poly(vinyl pyridinium chloride), Langmuir. 18 (2002) 9783-9791. doi:10.1021/la020503d.

[52] M.J. Armstrong, M.C. Carey, The hydrophobic-hydrophilic balance of bile salts. Inverse correlation between reverse-phase high performance liquid chromatographic mobilities and micellar cholesterol-solubilizing capacities., J. Lipid Res. 23 (1982) 7080. http://www.ncbi.nlm.nih.gov/pubmed/7057113. 